\title{
Identification of Aphasia using Natural Language Processing
}

\author{
Ananya Ananth Rao and Prof. Venkatesh S \\ Department of Electronics and Instrumentation Engineering, \\ $R V$ College of Engineering, Bangalore \\ ananyaananthrao.ei17@rvce.edu.in, venkateshs@rvce.edu.in
}

\begin{abstract}
Aphasia is a neurological disorder of language that precludes a person's ability to speak, understand, read or write in any language. By virtue of this disorder being inextricably connected to language, there is a vast potential for the application of Natural Language Processing (NLP) for the diagnosis of the disorder. This paper surveys the automated machine-learning based classification methodologies followed by an attempt to discuss a potential way in which an NLP backed methodology could be implemented along with its accompanying challenges. It is seen that the need for standardised technology-based diagnostic solutions necessitates exploration of such a methodology.
\end{abstract}

Keywords: Aphasia, Natural Language Processing (NLP)

\section{Introduction}

\subsection{Aphasia}

Aphasia is a condition that is caused by linguistic damage to regions of the brain. These areas are on the left side of the brain for the majority of people. Aphasia typically takes place quickly, commonly after a stroke or damage to the head, but also slowly due to brain tumours or degenerative neurologic disease. The disease affects linguistic expression and understanding, as well as reading and writing. Aphasia can occur with speech problems, including dysarthria or speech apraxia, also caused by brain injury [1]. In order to build a deeper understanding of Aphasia and its many types brought about by the region of the brain the damage is seen in, an understanding of language organisation in the brain is prudent. Spoken language enters the auditory cortex on both sides though data processing takes place on the left side of the brain. This information is processed in both the temporal lobe and the area known as the temporoparietal intersection (TPJ). The information enters the front lobe via two paths - the dorsal path that leads to speech production and the ventral path that leads to speech comprehension. Noises come that must be evaluated on all kinds of time frames. We must understand a noise, then put it in the form of phonemes together. After this we need to put them in syllables and place them in a syllable chain. The task of the ventral path is simple : to organise and produce a sound interpretation that is transmitted to the TPJ. The function is hence like a lexical interface. Here sensory information is procured and a meaning - a word or a semantic meaning - is attached to it. So we can speak through this lexical interface. Speech is the principal tool of human communication. Loss of speech or speech disruption is a serious hindrance to everyday life. Writing or reading, which can also be affected, is related to linguistic functions. There are therefore higher neurobiological impairments tied with aphasia. The aphasic symptoms that might be caused by injury to these networks can vary as the human brain consists of huge neural networks made of various functioning cerebral areas. Thus it is possible to distinguish the major forms of aphasia. There are two major types of aphasia: fluent 
and nonfluent, and within these groups there are a number of varieties. Syndromes represent a set of symptoms both empirical and statistically reliable that occur together [2].

- Broca's Aphasia (alternatively termed Motor or Expressive Aphasia): The difficulties of expressive linguistic functions are more obvious in Broca's aphasia than those of receptive language functions. With laboured, slow and impaired articulation, patients talk non-fluently. A key symptom is aggramatism, which means that the sentences are compressed to only a few words. However, Broca Aphasics' utterances are meaningful, and language understanding is less impacted.

- Wernicke's Aphasia (alternatively termed Sensory or Receptive Aphasia): In Wernicke's aphasia, both speech and articulation are properly produced. However, in contrast to the previous type, the phrases have little sense as the patient produces both literal paraphasias (change or omission of sounds within the words) and verbal paraphasias (use of incorrect words). Some patients produce sentences or words which are completely meaningless. Understanding and repeatability are seriously disrupted.

- Global Aphasia (or Total Aphasia): Global aphasia is a very serious linguistic problem that affects every language modality. It is often impossible to communicate.

- Anomic Aphasia: Though anomic patients produce spontaneous speech that is both fluent and grammatically accurate, the recovery of words in these patients is challenging. The difficulty in finding words can lead to breaks and circumlocutions. Understanding and repetition is quite normal.

- Conduction Aphasia: Although understanding seemingly is good, this condition renders the speaker struggling with spoken word repetition. It is typical to have literal paraphasias.

Brain areas whose damage leads to Broca's and Wernicke's aphasia have been displayed in Figure 1 below.

Even though most of those with aphasia tend to be older or middle-aged, anyone, including youngsters, can get it. Some 1 million people today suffer from aphasia in the United States, and almost 180,000 Americans get it every year, as per the National Aphasia Association. In India about 3 million people are affected [3]. Aphasia is caused by damage to one or more of the language areas of the brain. Most often, the cause of the brain injury is a stroke. Other causes could be severe head impacts, brain tumours, bullet injuries, brain infections, and progressive neurological illnesses such as Alzheimer's.

Aphasia is usually recognised first by a doctor who treats the person for his brain damage. Most people will get an MRI scan to confirm the presence of an abnormality in the brain and to pinpoint its accurate location. The doctor also usually assesses the 


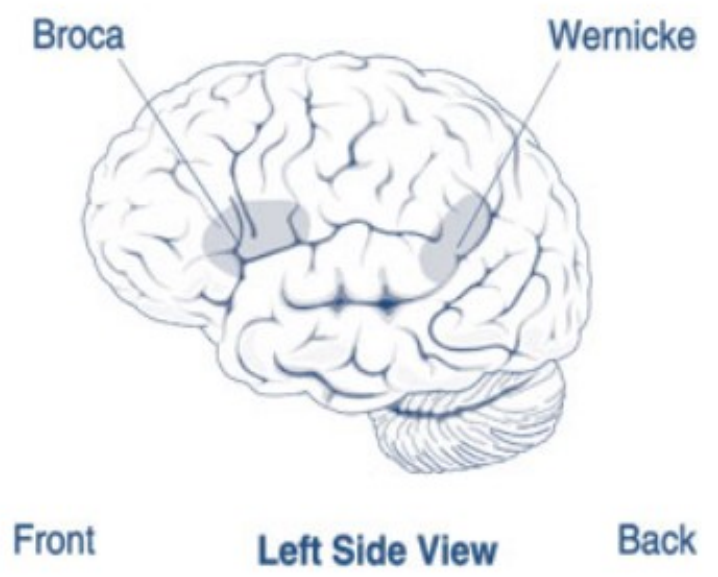

Fig.1. Broca's and Wernicke's aphasia [1]

capacity of the person to understand and generate language such as following orders, asking questions, identifying things and conducting a conversation. When the doctor detects aphasia, the patient may be referred to a pathologist who examines extensively the communication abilities of the person in communication. The ability of the person to speak, to express ideas, speak in social settings, understand, read and write are evaluated thoroughly.

One such prominent and popular test conducted for diagnosis purposes is the Aachen Aphasia Test (AAT) [4]. It yields a thorough assessment of Aphasia type and syndrome severity in a probabilistic manner following a thorough and lengthy - upto about eight hours for each person - testing process on various language modalities. The various AAT subtest include token test, spontaneous speech, repetition, written tests, confrontation naming and comprehension [2]. The duration of the test, presence of a certain amount of subjectivity in diagnosis due to it being dependent to quite an extent on the experience and judgement of the therapist, its human resource demands and lack of sensitivity to time-based changes necessitate the need for a more standardised, less timeand labour-intensive and more objective software based test. While still being based on the principles of AAT, it would increase the replicability of test results, enable personalisation of care irrespective of therapy location and pave way for at-home treatments.

After a brain injury, significant changes in the brain are observed that help it recover. Blood flow to the brain is disrupted sometimes and restored swiftly. When such a transitory ischemic attack occurs, language abilities can be reverted in a few hours or days. In the first few months, people with aphasia report substantial improvements, even without treatment, in their language and communication skills. However, some aphasia remains in many patients after this initial phase of recuperation. In these situations, speechlanguage therapy is utilised to assist patients in recovering their communication skills.

Studies show that language and communication abilities can continue to improve for many years and are at times accompanied by new activity in brain tissue near the damaged area. Some of the factors that may influence the amount of improvement include the cause of the brain injury, the area of the brain that was damaged and its extent, and the age and health of the individual. Therapy for this disorder is about increasing the ability of a person to communicate by enabling him or her to make maximum use of residual language abilities, restoring language skills, and learning other forms of communication, such as gestures, images or the use of electronic 
devices. Individual treatment focuses on the individual's particular requirements, while group therapy offers the chance to use new communication skills in a small group environment.Recent technology has equipped people with aphasia with new tools. "Virtual" speech pathologists enable patients to be flexibly and conveniently treated at home via a computer. An alternate means of communication for those who have trouble speaking might potentially be the use of speech generating software in mobile devices, for instance tablets. Patients with aphasia are becoming increasingly involved in activities such as book clubs, technology groups and theatre. These encounters enhance patients' confidence and social self-assurance and enhance their communication skills. Stroke clubs are accessible in most major cities along with regional support groups comprised of individuals who survived a stroke. These clubs may assist people and their families in adapting to the changes in life associated with stroke and aphasia. Family participation is often a vital part of aphasia, as it enables family members to discover the optimal manner of communicating with their loved one [1]

\section{Related Work}

Most of the literature surveyed puts forth methodologies that use the audio-speech aspect of Aphasia data. Audio clippings seem to be a more popular aspect of data chosen with pronunciation, speech quality and tone among others being explored. One applied selection with a transcript and low acoustical characteristics mainly aimed at obtaining a medical diagnosis, to distinguish two types of primary and progressive aphasia [5] while another utilised language and speech features to classify three broad frontotemporal lobar degeneration categories, which include progressive non-fluent aphasia [6]. There also are instances of utterance-level pronunciation and prosody scores prediction using speech samples [7][8][9] while another approach has been to identify and map to aphasia type and severity, vocal biomarkers such as automatisms, neologisms, loss of prosody and paragrammatism, from speech samples[10]. A method to carry out keyword spotting to recognise target words from phrases was proposed but this failed to pinpoint finer errors such as paraphasia [11][12]. Besides English, work with speech data in languages sans adequate datasets such as Greek and Kannada have also been attempted [13] [14]. Deep learning methods have been used for classifications with one of the first being used to detect paraphasia by detecting mispronunciations and various acoustic features like Phone Edit distance and Goodness of Pronunciation [15]. While supervised learning such as classification is most common, there is also an example of the development of an unsupervised method that reportedly outdoes preceding work using classification and does not depend on a specified target and posses the ability to be used on any language irrespective of data availability [16]. However, the method has so far been only tested on English but is still a noteworthy advancement in terms of approach.

In terms of theraputic approaches, there are three categories of computer systems developed for Aphasia diagnosis and treatment [17]. They are:

- Alternative and augmentative communication (AAC): Provides alternative ways of communication

- Computer-only treatment (COT): Provides therapist-independent support for Aphasia patients for the purpose of speech practice in the form of phone-based applications. This could mean patients being prompted into sentence building and recitation using predefined clauses with a touchscreen based interface,following which feedback and a target transcript would be presented [9]. The transcripts generated could also be used in the development of Automatic Speech Recognition (ASR) systems. 
- Computer-assisted treatment (CAT): Provides support to therapists engaged in Aphasia rehabilitative therapy. The technology analyses the patient's voice and gives an aphasia value during a conversational speech test [18] [19].

Different approaches for the automatic identifying of main progressive aphasia subtype were examined [20]. Two alternative feature detection algorithms were compared. The first technique they were trying to develop was a Welch test of functionality taken out of aphasic speech audio and transcription files compared with healthy speech. The findings were then ordered by the $\mathrm{p}$-values derived from the $\mathrm{t}$ test results and only the most relevant attributes were picked. Their second approach is based on the minimumredundancy-maximum-relevance technique (mRMR) [21]. The Naive Bayes probabilistic classification was contrasted with SVM and Random Forests. Their results reveal that, the combination of acoustic and transcript data improves aphasia subtype recognition as compared to only acoustic whose average accuracy was $74.05 \%$. Speech quality was another parameter assessed [22]. The parameters determining speech quality explored in the paper were clear-speech, which reflects the amount of speech clearly understood, fluidity and effort, which are indications of ease of speech with lesser long pauses and prosody. To improve generalisability and prevent overfitting, crossvalidation and feature selection using mRMR was implemented. Several commonlyused classifiers, including C4.5 Decision Tree, Logistic Regression, Naive Bayes, Random Forest, and Support Vector Machine were evaluated. Performance improves when there are less fine distinctions to make i.e, lesser classes to classify the data into. The results produced also address each of the four afore-mentioned speech quality parameters and may potentially be given to patients in real time thereby providing effective guidance.

All in all, the above works described make use of speech data in the form of audio clippings. It can be seen that there is a clear dearth of exploration of conducting a diagnosis based on the written tests that patients are made to undergo. One among the above comes close [19] wherein, following a 70:30 train-test split of data, the speech transcripts were converted into word vectors, labelled and given to a long short-term memory (LSTM) layer. This was followed by a densely connected layer with softmax activation function and categorical cross-entropy loss function. However, the peak accuracy was only at about $44.3 \%$ limiting its scope for deployment in actual real scenario. A better, thorough dataset of written tests with appropriate labelling could go a long way in improving results. This could be supplemented by the use of NLP for extractive summarization wherever applicable and sentiment analysis for classification. This aspect is explored in the next section.

\section{Proposed Potential Methodology}

In the presence of an exhaustive labelled dataset of written tests of Aphasia patients, sentiment analysis may be carried out to classify the presence or absence of aphasia. For larger bodies of text, an extractive text summarization may be performed as a first step.

\subsection{Natural Language Processing}

When machine learning models are used on data, these data tend to be in a format comprehensible to a those models. Directly analysing or classifying written human language itself, however, is not possible without some pre-processing and feature extraction. This is where NLP steps in. Natural Language Processing (NLP) is an algorithm that enables machines to process human language. They do so by performing certain operations on the words in the entirety of the dataset (corpus) 
and generate features that are a result of these operations. They include the following primary steps:

- Construction of Bag of Words: This process involves cleaning the data by removing punctuation and stopwords and then performing either or both stemming and lemmatization followed by vectorization. Stopwords are those words that tend to be relatively inconsequential to an analysis process by virtue of being too common without contributing to any significant information. Words like 'i', 'me', 'my', 'myself', 'we', 'our', 'ours', 'ourselves', 'you' and 'your' are some examples of stopwords. Stemming and lemmatization are similar to one another in that involves cutting various forms of a word down to a single common word that tends to be the root word. While stemming does this without regard to meaning or grammar, lemmatisation does this with consideration to grammar, producing an actual, meaningful root word. The decision between using stemming or lemmatization depends on the specific use case. Following this, vectorisation is carried out. This involves constructing a matrix - preferably sparse - of unique words in the corpus indexed by the number of documents in the corpus. For every document, therefore, a $1 \times n$ vector would be produced where $n=$ number of unique words within the corpus.

- TF-IDF: Term Frequency is the importance of the term within that document while Inverse Document Frequency is the importance of the term in the corpus [23]. This is done to extract the significance of each term within the corpus and assign a higher weightage to those terms that are statistically more important in identifying a particular target class. Word that occur more often in a document would be potentially important to the document. However, if the same term occurs often throughout the entire corpus then it would not be as useful as a distinguishing feature. The TF-IDF calculation takes care of these two considerations while highlighting and yet balancing out the importance of terms in the document. The equation is as given below

[23] -

$$
\mathbf{w}_{x, y}=\mathrm{tf}_{x, y} \times \log \left(\frac{N}{d f_{x}}\right)
$$

Where:

$\mathrm{tf}_{\mathrm{x}, y}=$ frequency of $\mathrm{x}$ in $\mathrm{y} \mathrm{df}_{\mathrm{x}}=$ number of

documents containing $x \mathrm{~N}=$ total

number of documents

- Model building and deployment: This is the final step and involves using the features extracted from the corpus to build a classification model. The necessity of this step would depend on the use case. It is, however, for most popular applications of NLP, a very common end step. While any standard, wellperforming machine learning model may be used, Naive Bayes is one of the most commonly used classifier for NLP applications.

NLP finds application in tasks such as sentiment analysis, language translation, information extraction and so on [24].

\subsection{Sentiment Analysis}

The process of sentiment analysis is carried out to glean the underlying sentiment of a body of text. It is most popularly used by business organisations to analyse customer feedback and queries in order to gather insights on how to better their business strategies [25] [26] [27]. The code used to build the sentiment analyser, however, can be used to classify between non-aphasia and aphasia subjects since the very essence of a sentiment analyser is to classify between the various given labelled 
classes as per the steps mentioned in the previous subsection and as shown in Figure 2 below.

\subsection{Text Summarization}

Text summarization is another popular application of NLP that is used to mitigate the woes of going through lengthy text bodies and like sentiment analysis, largely used by business organisations to summarize the content of customer reviews. For aphasia based written tests, text summarization can be an optional step in case of lengthy text bodies.

There are several ways to classify text summarization. Of relevance here are the types of text summarization based on type of type of output [28]:

- Extractive Text Summarization: In this, a condensed version of the text body is given by extracting sentences of importance from the text itself. No new words are generated and all words in the summary are extracted from the text itself and is shown in Figure 3 below.

- Abstractive Text Summarization: In this the condensed version is given by generating a set of new sentences. These sentences are generated by learning from the input text body and forming new sentences that convey the same meaning and is seen in Figure 4 below.

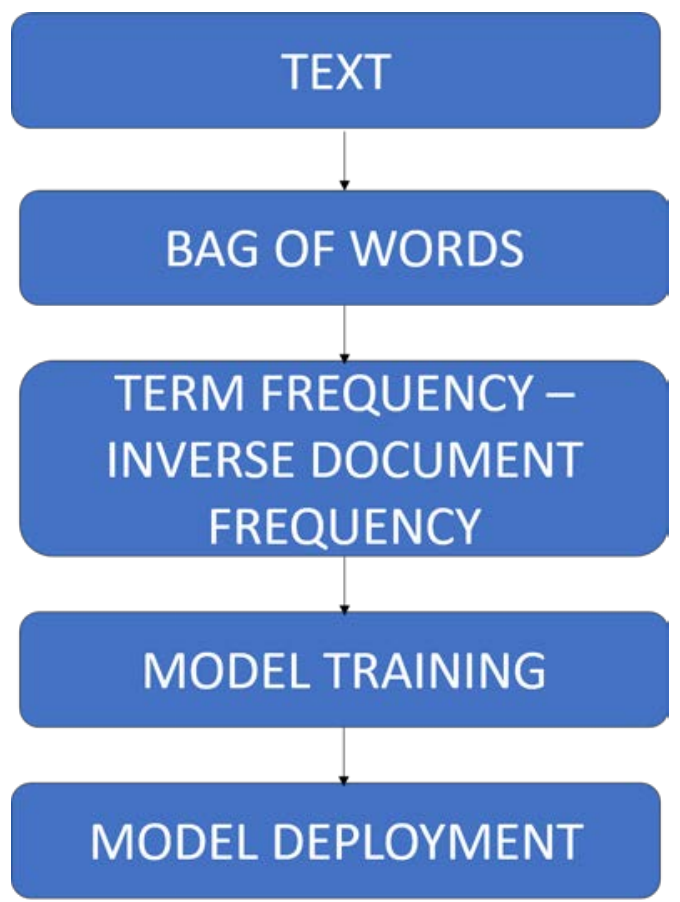

Fig.2. Typical NLP based methodology for sentiment analysis 


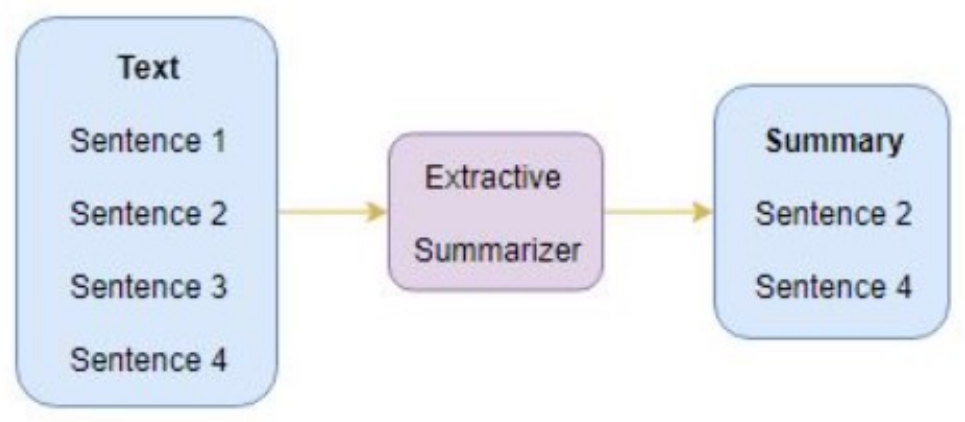

Fig.3. Extractive Text Summarization [29]

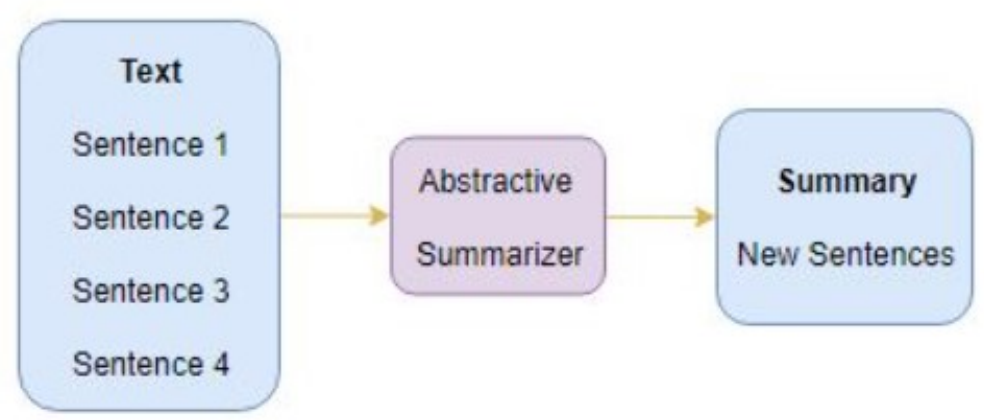

Fig.4. Abstractive Text Summarization [29]

A procedure using seq2seq model with the LSTM and attention mechanism has been found to provide the highest accuracy [28]. Any kind of sequential information is a good candidate for the application of seq2seq model which consists of an encoder and decoder as its two main components as seen in Figure 5. This kind of architecture is useful for an input-output combination of varying length. For input sequences of greater lengths, the above-mentioned architecture falls short and requires the attention mechanism to step in and augment the process. The attention mechanism helps inflate the importance of parts of the input instead of its entirety.

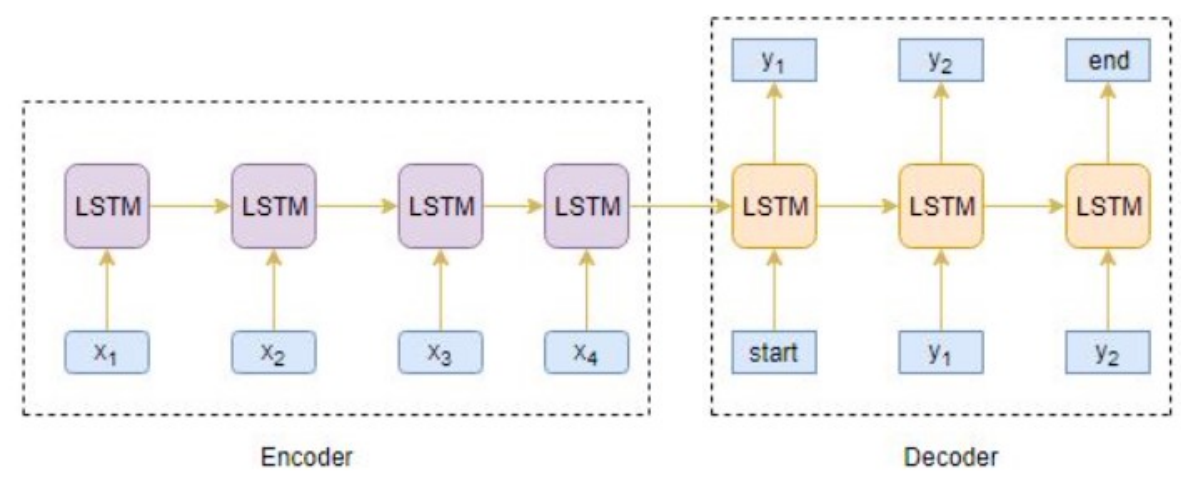


Fig.5. Conventional seq2seq model [29]

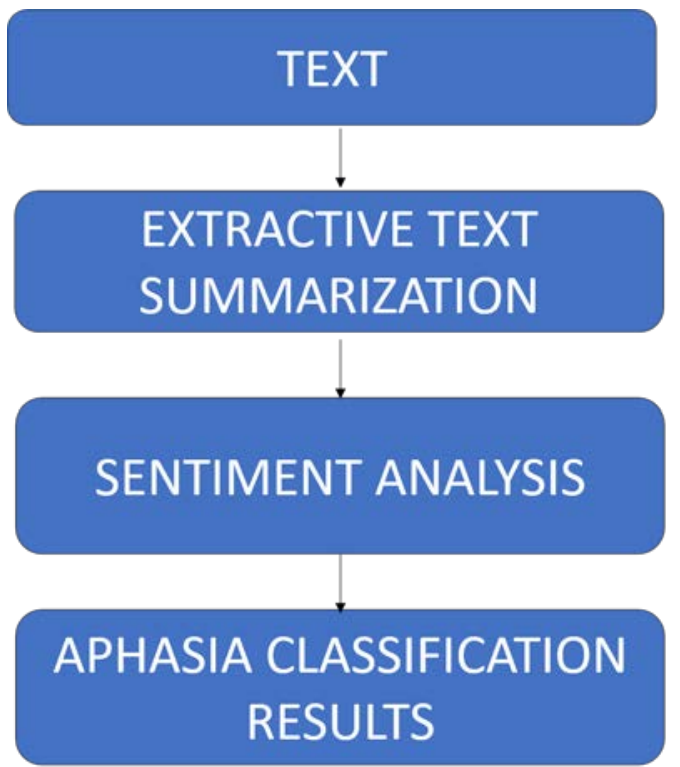

Fig.6. Proposed potential methodology

\section{Conclusion and future scope}

Figure 6 shows the final version of the proposed methodology. This consists of the optional step of extractive text summarization followed by a mandatory step of sentiment analysis. It would thus bring about an efficient classification methodology based on the written language tests undergone by Aphasia patients.

There is a very striking prevalence of speech data usage for machine learning based classification systems. The exhaustive exploration done by various researchers in this area witnesses a combination of some or all of signal processing, feature extraction and supervised as well as unsupervised learning methodologies. This is accompanied by a clear gap in evaluations of the writing capabilities of aphasia patients thereby necessitating a thorough exploration of the same.

It is seen that the lack of availability of an exhaustive, labelled dataset of written tests of Aphasia patients precludes the evaluation of the effectiveness of the proposed method. However, sentiment analysis and text summarization algorithms are widely popular and may be easily tweaked to suit the use case in question. The use case may also be extended to include classification between the various subtypes of aphasia itself.

In a world that is fast moving towards technology-based innovations in every walk of life, the reach of technology in healthcare has been comparatively limited. The need for and popularity of standardization, personalisation and replicability in healthcare has also risen exponentially of late. There is thus a vast scope for technology-driven impact in this sector including diagnosis methods, personalised treatments and therapy. This exploration of technology-assisted methods based on pre-existing well-established and researched protocols shows immense promise. Aphasia is one such area that is in need of efficient, consistent and reliable technological innovation. The NLP based methodology presented above is one such way in which positive impact could be brought about and therefore merits further exploration. 


\section{References}

1. https://www.nidcd.nih.gov/health/aphasia

2. https://citeseerx.ist.psu.edu/viewdoc/download?doi=10.1.1.4.4880\&rep=rep1\&type=pdf

3. K. R. Jothi, S. S. Sivaraju and P. J. Yawalkar, "Al based Speech Language Therapy usingSpeech Quality Parameters for Aphasia Person: A Comprehensive Review," 2020 4th International Conference on Electronics, Communication and Aerospace Technology (ICECA), 2020, pp. 1263-1271, doi: 10.1109/ICECA49313.2020.9297591. =rep1type=pdf

4. Huber, W., Poeck, K., and Springer, L. (2013). Klinik und Rehabilitation der Aphasie: eineEinfuhrung $f$ " ur Thera-peuten, Angehorige und Betroffene“ . Georg Thieme Verlag.

5. Kathleen Fraser, Frank Rudzicz, and Elizabeth Rochon. 2013. Using text and acoustic features to diagnose progressive aphasia and its subtypes.

6. Bart Peintner, William Jarrold, Dimitra Vergyri, Colleen Richey, Maria Luisa GornoTempini, and 19 Jennifer Ogar. 2008. Learning diagnostic models using speech and language measures. Conference proceedings : ... Annual International Conference of the IEEE Engineering in Medicine and Biology Society. IEEE Engineering in Medicine and Biology Society. Conference, 2008:4648-51.

7. Duc Le, Keli Licata, Elizabeth Mercado, Carol Persad, and Emily Mower Provost. 2014. Automatic analysis of speech quality for aphasia treatment. pages 4853-4857.

8. Duc Le and Emily Mower Provost. 2015. Modeling pronunciation, rhythm, and intonationfor automatic assessment of speech quality in aphasia rehabilitation

9. Duc Le, Keli Licata, Carol Persad, and Emily Mower Provost. 2016. Automatic assessmentof speech intelligibility for individuals with aphasia. IEEE/ACM Transactions on Audio, Speech, and Language Processing, 24:1-1.

10. C. Kohlschein, M. Schmitt, B. Schuller, S. Jeschke and C. J. Werner, "A machine learning" based system for the automatic evaluation of aphasia speech," 2017 IEEE 19th International Conference on e-Health Networking, Applications and Services (Healthcom), 2017, pp. 1-6, doi: 10.1109/HealthCom.2017.8210766.

11. Alberto Abad, Anna Pompili, Angela Costa, and Isabel Trancoso. 2012. Automatic wordnaming recognition for treatment and assessment of aphasia. 13th Annual Conference of the International Speech Communication Association 2012, INTERSPEECH 2012, 2.

12. Alberto Abad, Anna Pompili, Angela Costa, Isabel Trancoso, Jos Fonseca, Gabriela Leal,LuisaFarrajota, and Isabel Martins. 2013. Automatic word naming recognition for an online aphasia treatment system. Computer Speech Language, 27:12351248.

13. SpyridoulaVarlokosta, SpyridoulaStamouli, Athanasios Karasimos, Georgios Markopoulos, Maria Kakavoulia, Michaela Nerantzini, et al., A Greek Corpus of Aphasic Discourse: Collection Transcription and Annotation Specifications, 2016.

14. R. Shenoy, S. Nayak, M. K. Hegde, N. Kini, P. P. Kundapur and G. Krishnan, "Development of an android application in kannada to enhance picture naming skills in persons with aphasia," 2017 International Conference on Advances in Computing, Communications and Informatics (ICACCI), 2017, pp. 2134-2140, doi: 10.1109/ICACCI.2017.8126161.

15. Duc Le, Keli Licata, and Emily Mower Provost. 2017. Automatic paraphasia detection fromaphasic speech: A preliminary study. In Interspeech, pages 294-298.

16. https://www.aclweb.org/anthology/2020.acl-srw.3.pdf

17. Katz, R. C. (2010). Computers in the treatment of chronic aphasia. In Seminars in speechand language, volume 31, pages 034-041. Published by Thieme Medical Publishers.

18. Kohlschein, C., Schmitt, M., Schuller, B., Jeschke, S., and Werner, C. J. (2017). A machinelearning based system for the automatic evaluation of aphasia speech. In 2017 IEEE 19th International Conference on e-Health Networking, Applications and Services (Healthcom).

19. https://aphasia.talkbank.org/publications/2018/Kohlschein18.pdf

20. Fraser, K. C., Rudzicz, F., and Rochon, E. (2013). Using text and acoustic features to diagnose progressive aphasia and its subtypes. In INTERSPEECH, pages 2177-2181.

21. Peng, H., Long, F., and Ding, C. (2005). Feature selection based on mutual information criteria of max-dependency, max-relevance, and min-redundancy. IEEE Transactions on pattern analysis and machine intelligence, 27(8):1226-1238. 
22. https://web.eecs.umich.edu/ emilykmp/EmilyPapers/Le2014ICASSP2014.pdf

23. https://towardsdatascience.com/tf-term-frequency-idf-inverse-document-frequencyfromscratch-in-python-6c2b61b78558

24. https://towardsdatascience.com/natural-language-processing-nlp-for-machinelearningd44498845d5b

25. Y. A. Solangi, Z. A. Solangi, S. Aarain, A. Abro, G. A. Mallah and A. Shah, "ReviewonNatural Language Processing (NLP) and Its Toolkits for Opinion Mining and Sentiment Analysis," 2018 IEEE 5th International Conference on Engineering Technologies and Applied Sciences (ICETAS), 2018, pp. 1-4, doi: 10.1109/ICETAS.2018.8629198.

26. M. R. Hasan, M. Maliha and M. Arifuzzaman, "Sentiment Analysis with NLP on Twitter Data," 2019 International Conference on Computer, Communication, Chemical, Materials and Electronic Engineering (IC4ME2), 2019, pp. 1-4, doi: 10.1109/IC4ME247184.2019.9036670.

27. Y. Woldemariam, "Sentiment analysis in a cross-media analysis framework," 2016 IEEE International Conference on Big Data Analysis (ICBDA), 2016, pp. 1-5, doi: 10.1109/ICBDA.2016.7509790.

28. R. Boorugu and G. Ramesh, "A Survey on NLP based Text Summarization for SummarizingProduct Reviews," 2020 Second International Conference on Inventive Research in Computing Applications (ICIRCA), 2020, pp. 352-356, doi: 10.1109/ICIRCA48905.2020.9183355.

29. https://www.analyticsvidhya.com/blog/2019/06/comprehensive-guide-textsummarizationusing-deep-learning-python/ 